\title{
The waterlogging of floating objects
}

\author{
DOMINIC VELLA AND HERBERT E. HUPPERT \\ Institute of Theoretical Geophysics, Department of Applied Mathematics and Theoretical Physics, \\ University of Cambridge, Wilberforce Road, Cambridge, CB3 0WA, UK
}

(Received 3 January 2007 and in revised form 6 June 2007)

We consider the dynamical processes by which a dense, porous object floating in a body of liquid becomes waterlogged and sinks. We first generalize the classic model of capillary rise in a porous medium to present an analytically tractable model of the process, which is valid for objects that are very shallow compared to their horizontal extent. We find an analytical expression for the time taken for the object to sink under this approximation. We use a series of boundary integral simulations to show that decreasing the horizontal extent of the object decreases the time taken to sink. We find that the results of these numerical simulations are in good quantitative agreement with a series of laboratory experiments. Finally, we discuss the implications of our work for pumice fragments, often found floating in open water after a volcanic eruption, occasionally even supporting plant, animal and human remains.

\section{Introduction}

Very porous rocks, such as pumice, are able to float on water when dry because their bulk density is below that of water. They do not remain dry for long, however: a combination of capillary suction and the hydrostatic pressure in the exterior liquid forces water to flow into the rock. This influx of water increases the bulk density of the rock and ultimately may cause it to sink.

The waterlogging process can be extremely drawn out for fragments of pumice, which are often found floating in oceans many months or even years after a volcanic eruption (Whitham \& Sparks 1986). During this time, the fragments follow ocean currents, which suggests that tracking their progress might yield information on the speed and direction of these currents (Bryan et al. 2004). The sediments formed once pumice fragments sink also have interesting features that have been noted by sedimentologists (Manville et al. 1998). In these and other applications, it is important to understand the time taken for the pumice to become sufficiently waterlogged that it sinks. Here we address this question by formulating and solving a fluid mechanical model of the waterlogging of a floating object and consider the geophysical implications of our results.

We begin by discussing the theoretical formulation in terms of the flow into a floating, porous body. Using a one-dimensional formulation, we are able to derive an analytical expression for the level at which the body floats in the liquid as a function of time. We also obtain an analytical expression for the time taken to sink as a function of the various material properties of the body. We then consider the two-dimensional situation. This cannot be solved analytically and so we introduce a boundary integral formulation, which we solve numerically. We discuss the results of these numerical simulations and compare them to a series of laboratory experiments. Finally, we describe briefly some of the implications of our work for the saturation 
(a)

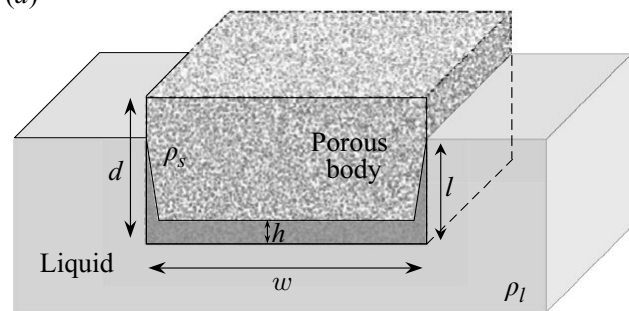

(b)

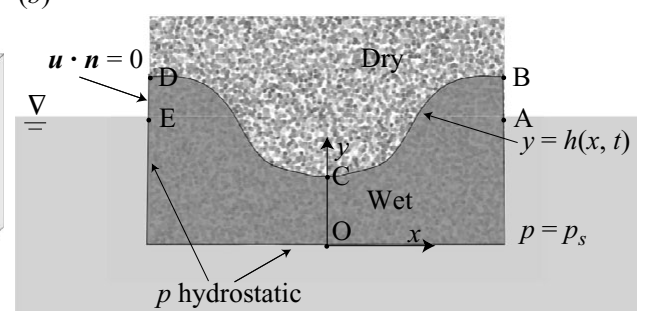

FiguRE 1. (a) Schematic illustration of a porous block floating in a liquid. Due to the combined action of surface tension and hydrostatic pressure, the liquid is imbibed by the porous medium and so the exterior level of the liquid, $l$, rises. (b) Cross-section through the porous block illustrating the boundary conditions.

of pumice, which often floats (temporarily) on bodies of open water after a volcanic eruption.

\section{Theoretical formulation}

Consider a hydrophilic porous body with solid density $\rho_{s}$ and constant, isotropic porosity $\phi$. If this is placed in air (of negligible density and viscosity) on the surface of a liquid with density $\rho_{l}>\rho_{s}(1-\phi)$ then the body will initially float, as shown in figure 1. However, the combined actions of the interfacial tension, $\gamma$, and the hydrostatic pressure in the liquid outside the body will force the liquid to impregnate the porous body. If $\rho_{s}>\rho_{l}$ and the pores are all connected then this process will ultimately cause the object to sink. We are interested in the dynamical processes by which the object becomes waterlogged, and the time at which it will sink.

\subsection{Governing equations}

In general, regions of the porous medium may only be partially saturated by the wetting liquid (water), leaving some residual air saturation. We should therefore compute the evolution of the saturation field (Bear 1988). However, in capillary absorption the width of the partially saturated region is typically a constant fraction of the width of the wetted region (Philip 1969). By observing the capillary absorption of coloured food dye into several pieces of pumice, we estimate that this partial saturation region (signified by variations in coloration) is typically $\lesssim 5 \%$ of the overall wetted region. Since this region is relatively limited in these rocks, we expect the effects of partial saturation on their time afloat to be small. We shall, therefore, use a sharp interface model in which the free surface separates regions of the object that are fully dry and fully wet. The flow of liquid into the porous body is then Darcy flow (Bear 1988) in a gravitational field. According to Darcy's law, the liquid velocity within the porous medium, $\boldsymbol{u}$, is linearly related to the pressure gradient by

$$
\boldsymbol{u}=\frac{k}{\phi \mu}\left(-\nabla p+\rho_{l} \boldsymbol{g}\right),
$$

where $\mu$ is the dynamic viscosity of the liquid and $k$ is the permeability (assumed uniform) of the porous body. The incompressibility of the liquid requires that $\nabla \cdot \boldsymbol{u}=0$ so that the pressure in the liquid satisfies

$$
\nabla^{2} p=0 .
$$


From (2.1) it is clear that the pressure $p$ completely specifies the flow within the porous medium. The problem therefore reduces to solving Laplace's equation (2.2) with suitable boundary conditions. We assume that the pressure distribution in the liquid outside the floating body is purely hydrostatic because sinking typically occurs very slowly. The pressure along the boundary of the body in contact with the exterior liquid (AOE in figure $1 b$ ) is therefore specified solely by the position of the object in the liquid.

The boundary condition along the air-liquid interface is more complicated. Where the interface lies within the porous medium (BCD in figure $1 b$ ), we assume that there is a constant pressure jump across the interface, $\Delta p=\gamma \kappa$, where $\kappa$ is the pore-scale curvature. Taking atmospheric pressure as the zero pressure datum, the boundary condition on this interface is therefore $p=p_{s}$, a constant. Where the airliquid interface coincides with the edges of the porous medium itself (AB and DE in figure $1 b$ ), the pore-scale meniscus curvature is ill-defined. Here the capillary pressure acts against the hydrostatic head that would tend to drive the fluid to flow out of the block and so there is no fluid flux through these boundaries, i.e. $\boldsymbol{u} \cdot \boldsymbol{n}=0$ where $\boldsymbol{n}$ is the normal to the edge of the body. These interfaces are called capillary exposed faces in the literature (Bear 1988, §7.1.9). The boundary conditions for this problem are summarized in figure $1 b$.

The level of the floating body is determined by the assumption that the vertical force balance on the body is satisfied instantaneously. Archimedes' principle applied to the floating body requires that the weight of exterior liquid displaced by the body must be equal to the weight of the body including any imbibed liquid. However, it is difficult to express this condition mathematically for general body shapes.

Our main focus is on determining the time taken for a given porous body to become waterlogged to the point where it is no longer able to float and therefore sinks. We expect this process to occur on the time scale, $t^{*}$, over which the liquid flows a distance into the porous medium comparable to its characteristic depth, $d$. From (2.1) the typical fluid speed is $u^{*}=k \rho_{l} g / \phi \mu$ suggesting that we should define

$$
t^{*} \equiv d \phi \mu /\left(k \rho_{l} g\right) \text {. }
$$

We shall use this characteristic time to non-dimensionalize time, $t$, taking $T=t / t^{*}$. Similarly, we use the characteristic depth $d$ to non-dimensionalize lengths, taking $X=$ $x / d, Y=y / d$ and so on. Finally, we introduce a dimensionless pressure $P=p / \rho_{l} g d$ (again measured relative to the atmospheric pressure). Taking $\Pi=-p_{s} / \rho_{l} g d$, the constant pressure along the air-liquid interface due to capillarity is $P=-\Pi$ (the minus sign signifying that, for the hydrophilic materials of interest here, capillarity acts to suck liquid into the porous medium). We note that for the experiments presented in $\S 4, \Pi=O(1)$ : the capillary suction pressure and the external hydrostatic pressure are typically of comparable magnitude.

With this formulation of the problem we now turn to a one-dimensional model, a modification of the classic analysis of capillary rise in a porous medium given by Washburn (1921). As in that analysis, we are able to solve the problem analytically in this limit.

\subsection{A one-dimensional model}

We begin by considering a one-dimensional model in which liquid can only flow in through the base of the object (the line $Y=0$ in figure $1 b$ ). This corresponds to the limit in which the object is very shallow in comparison to its horizontal extent. In the onedimensional case, the air-liquid interface remains planar so that its height above the 
base is $H(X, T) \equiv H(T)$. Similarly, the pressure within the porous body must only be a function of the height, $Y$, from the base of the body and time $T$. The pressure will also satisfy Laplace's equation (2.2) with boundary conditions $P(0)=L$ and $P(H)=-\Pi$ corresponding to hydrostatic pressure in the exterior liquid at $Y=0$ and the capillarityinduced pressure at the air-liquid interface, respectively. We therefore find

$$
\mathrm{d} P / \mathrm{d} Y=-(\Pi+L) / H .
$$

Substituting this pressure gradient into Darcy's law (2.1) and equating the liquid velocity to the rate at which the interface moves through the body, we find that

$$
\mathrm{d} H / \mathrm{d} T=(\Pi+L) / H-1 .
$$

This ordinary differential equation for the motion of the air-liquid interface through the porous medium is dependent on both the height of the interface from the base, $H$, and the submerged depth of the body, $L$. These two quantities are, however, related through the condition of vertical force balance, which here simplifies to

$$
L=\phi H+D(1-\phi)
$$

in which $D \equiv \rho_{s} / \rho_{l}>1$ is the density ratio of solid to liquid. Eliminating $L$ from (2.5) using (2.6) and integrating the result subject to $H(0)=0$, we find that

$$
(1-\phi) T=-H-\Pi_{e} \log \left(1-H / \Pi_{e}\right)
$$

where

$$
\Pi_{e} \equiv D+\Pi(1-\phi)^{-1}
$$

is the effective non-dimensional pressure driving the waterlogging of the body. Note that the density of the object enters (2.7) only through the modified driving pressure in (2.8). This is a result of the vertical force balance condition, which requires that the pressure at the base increase linearly with the solid density in order to allow the object to float.

It is not possible to invert (2.7) to give the immersed depth of the object, $H$, in terms of elementary functions of $T$. However, we may write

$$
H=\Pi_{e}\left\{1+W\left[-\exp \left(-1-\frac{1-\phi}{\Pi_{e}} T\right)\right]\right\},
$$

where $W(x)$ is the Lambert $W$ function, defined as the solution of $x=W(x) \exp [W(x)]$ (see Corless et al. 1996, for example). In fact, the form of (2.7) is extremely convenient for determining the time, $T_{s}$, taken for the body to become waterlogged and sink: sinking occurs when the exterior free surface coincides with the top edge of the body, i.e. when $L=1$. From (2.6) we therefore have that $H\left(T_{s}\right)=D+(1-D) / \phi$, which may be substituted directly into (2.7) to give an explicit expression for $T_{s}$ in terms of $D, \phi$ and $\Pi_{e}$. Using (2.8) to eliminate $D$ in favour of $\Pi$, this expression simplifies to

$$
T_{s}=\frac{\Pi_{e}-\Pi-1}{\phi(1-\phi)}-\frac{\Pi_{e}}{1-\phi}\left[1+\log \left(\frac{\Pi_{e}-\Pi-1}{\Pi_{e} \phi}\right)\right] .
$$

\section{Numerical simulations}

In the one-dimensional model, we have assumed that liquid can only flow in through the base of the floating object and flows only vertically. In general, the flow through 
the sides of the body could also be considerable and cause the object to reach the sinking density more quickly. We now consider how much of an effect this is.

For simplicity, we consider a two-dimensional body with rectangular cross-section, as shown in figure 1 . The body has width $w$ and depth $d$ as in figure 1(a). In nondimensional terms the porous medium occupies the region $|X| \leqslant \alpha / 2,0 \leqslant Y \leqslant 1$, where $\alpha \equiv w / d$ is the aspect ratio of the body. The air-liquid interface is given by $Y=$ $H(X, T)$ and moves through the body according to the kinematic boundary condition

$$
\frac{\mathrm{D} H}{\mathrm{D} T}=\left.\boldsymbol{U} \cdot \boldsymbol{e}_{y}\right|_{Y=H},
$$

where $\boldsymbol{e}_{y}$ is the unit normal in the vertical direction and $\boldsymbol{U}$ is given by the dimensionless version of Darcy's law (2.1). The evolution of the air-liquid interface therefore depends on the pressure in the liquid and thus on the level, $L$, at which the body floats in the liquid. As the liquid infiltrates the floating body, $L$ increases according to the vertical force balance condition, which requires that

$$
L=D(1-\phi)+\frac{\phi}{\alpha} \int_{-\alpha / 2}^{\alpha / 2} H \mathrm{~d} X .
$$

As soon as $L>1$, the body sinks because it is then more dense than the surrounding liquid. What interests us is the time taken for the body to reach the critical value $L=1$.

In this two-dimensional problem, the air-liquid interface does not remain planar and the associated free-boundary problem cannot be solved analytically. The sinking time cannot, therefore, be determined analytically and we resort to a numerical method to calculate the dynamics of liquid motion in the body and thereby calculate $T_{s}$. We now discuss this numerical method in more detail.

\subsection{Numerical method}

We used a boundary integral method to solve Laplace's equation (2.2) for the pressure $P$ in the porous medium. Our method is based on a boundary integral method used to study two-dimensional potential flow problems (see Tsai \& Yue 1996, for a review of these methods). More details are given by Greenhow et al. (1982). The connection to two-dimensional potential flows arises upon introducing a velocity potential $\Phi \equiv-Y-P$ so that $\boldsymbol{U}=\nabla \Phi$. It is then natural to introduce the corresponding streamfunction $\Psi(X, Y ; T)$. Both $\Phi$ and $\Psi$ are harmonic functions and so the complex potential $\beta(Z ; T) \equiv \Phi(X, Y ; T)+\mathrm{i} \Psi(X, Y ; T)$ is analytic within the wetted porous medium (where $Z=X+\mathrm{i} Y$ ). By Cauchy's theorem, therefore,

$$
\int_{\mathscr{C}} \frac{\beta}{Z-Z_{k}} \mathrm{~d} Z=0,
$$

where $\mathscr{C}$ is the closed contour bounding the wetted porous medium (ABCDEOA in figure $1 b$ ) and $Z_{k}$ is a point outside the contour $\mathscr{C}$.

At an instant of time, the pressure on most of the air-liquid interface and on the portion of perimeter that is in contact with the exterior fluid is given by the configuration of the body. The velocity potential $\Phi$ is therefore known at these positions on the contour $\mathscr{C}$. On the small portions of the air-liquid interface that coincide with the boundary of the porous body (the capillary exposed faces), $\Phi$ is unknown since the pressure there is unknown. However, because there is no flux through these faces, they are streamlines and we may set $\Psi=0$ along these portions of $\mathscr{C}$. Therefore either the real or imaginary part of $\beta$ is known for every portion of 
$\mathscr{C}$. Upon discretizing the contour $\mathscr{C},(3.3)$ gives rise to a series of Fredholm integral equations of the second kind for the unknown parts of $\beta$. These equations may then be solved numerically (as discussed by Greenhow et al. 1982) to give the unknown part of $\beta$ at each point of the contour $\mathscr{C}$. The complex potential $\beta$ is therefore known all around $\mathscr{C}$ at a given instant of time.

From complex potential theory, the velocity $(u, v)$ of the fluid within the porous medium satisfies $u-\mathrm{i} v=\mathrm{d} \beta / \mathrm{d} Z$ (Batchelor 1967). The kinematic boundary condition (3.1) for the motion of points on the air-liquid interface may therefore be written as

$$
\mathrm{D} Z / \mathrm{D} T=(\mathrm{d} \beta / \mathrm{d} Z)^{*},
$$

where * denotes the complex conjugate. The derivative in (3.4) is calculated numerically using a second-order central-difference scheme. The position of points on the air-liquid interface is then stepped forward in time using the fourth-order RungeKutta method. The points are regularly redistributed according to the smoothing scheme proposed by Longuet-Higgins \& Cokelet (1976) to suppress an unphysical interfacial instability. The new value of $L$, the level at which the body floats in the liquid, is calculated using (3.2). This method is repeated marching forward in time, until $L=1$ at some time $T=T_{s}$.

Our simulations typically used 100 interfacial nodes and a simple variable-time-step routine. The convergence of our scheme was tested by varying the number of nodes and the time step; the results presented here are accurate to $\$ 1 \%$. We validated our numerical scheme using Washburn's (1921) analytical result for capillary absorption into a fixed porous medium and our own result (2.9) for the limit of shallow floating objects.

\subsection{Results}

The non-dimensional time to sink depends on the four non-dimensional parameters in the problem: the density ratio $D$; porosity $\phi$; aspect ratio $\alpha$; and the strength of surface tension $\Pi$. In the case of pumice clasts the first two of these parameters are reasonably constant with $D \approx 2.3$ and $\phi \approx 0.8$ (Whitham \& Sparks 1986). In the numerical results presented here, therefore, we have held these two parameters constant and varied only the aspect ratio $\alpha$ and the strength of surface tension $\Pi$.

The time taken to sink, $T_{s}$, as calculated by our boundary integral simulations is plotted in figure 2 as a function of $1 / \Pi$ for different values of the aspect ratio $\alpha$. It is convenient to use $1 / \Pi=-\rho_{l} g d / p_{s}$ as the independent variable in this graph since, with all else held constant, increasing $1 / \Pi$ corresponds to increasing the size of the body. For $1 / \Pi \ll 1$ figure 2 shows that $T_{s} \sim 1 / \Pi$, which in dimensional terms indicates that $t_{s} \sim d^{2}$.

The numerical results show that, as expected, the finite aspect ratio of a floating porous body decreases the value of $T_{s}$ (compared to the one-dimensional limit considered earlier). This is because there is an additional boundary through which liquid can flow into the body. The one-dimensional model shows that for given material properties there is a maximum non-dimensional time to sink, $T_{\max }$, which is given by taking the limit $\Pi \rightarrow 0$ in (2.10). For $1 / \Pi \gg 1$, therefore, we must have $T_{s} \rightarrow \beta$, where $\beta<T_{\max }$ is a constant. In dimensional terms this shows that the time to sink $t_{s} \sim d$ for $\Pi \ll 1$. Summarizing the results for $\Pi \gg 1$ and $\Pi \ll 1$ we have:

$$
t_{s} \sim \begin{cases}d^{2}, & d \ll\left|p_{s}\right| / \rho_{l} g, \\ d, & d \gg\left|p_{s}\right| / \rho_{l} g .\end{cases}
$$




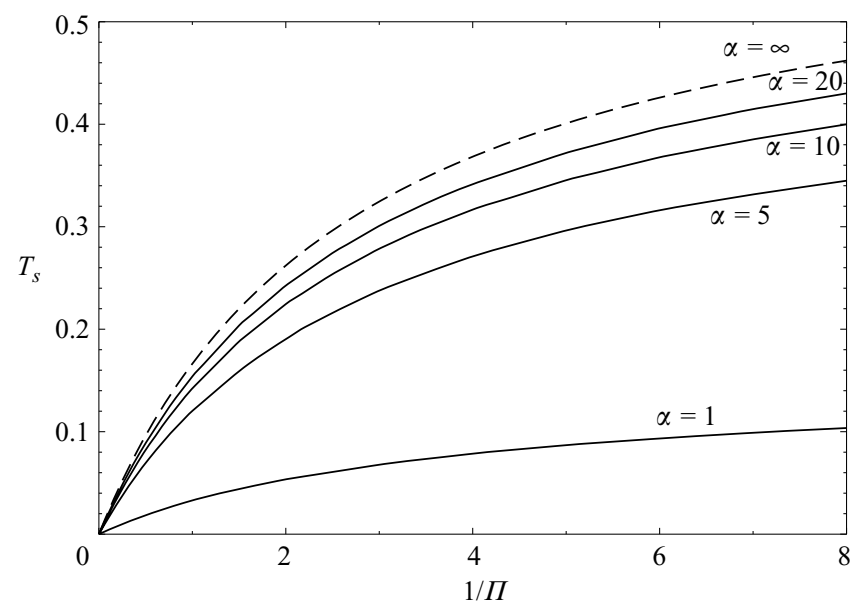

FiguRE 2. Numerical results for the non-dimensional time to sink, $T_{s}$, as a function of the strength of surface tension, $\Pi$, for various values of the aspect ratio of the object. The dashed line shows the analytical prediction of the one-dimensional model (2.10), corresponding to the limit $\alpha=\infty$. Here $D=2.3$ and $\phi=0.8$, as is typical of pumice (Whitham \& Sparks 1986).

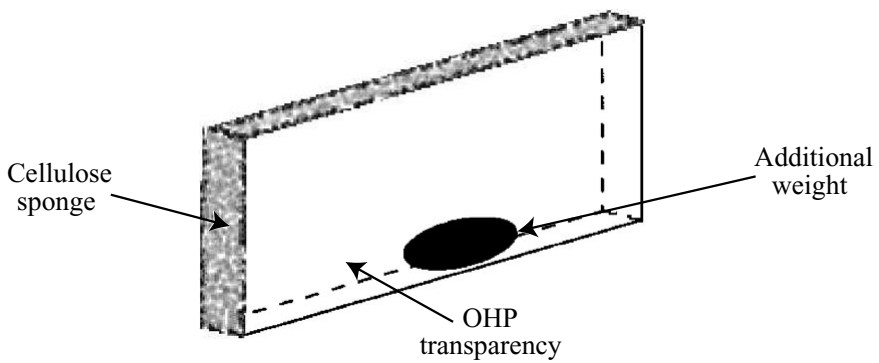

FIgURE 3. Schematic illustration of the sponge sheets used in our experiments. Typically the sponge was around $1 \mathrm{~cm}$ thick with a cross-section of $10 \mathrm{~cm} \times 5 \mathrm{~cm}$.

\section{Experimental results}

We conducted a series of simple experiments to directly test our theoretical results. In these experiments the porous medium used was hydrophilic cellulose sponge (Sydney Heath and Son). Sponge was chosen rather than pumice because it appeared to be isotropic, was easy to cut into different sized pieces and could be dried quickly between runs. The sponge has only a limited region of partial saturation $(<5 \%)$.

The porosity of the sponge was measured to be $\phi \approx 0.77$. Its permeability was measured in a dynamic capillary rise experiment: the sponge was vertically clamped and the capillary rise of dyed water through it was recorded using a CCD camera. The measured height of capillary rise as a function of time agrees well with the theoretical prediction of Washburn (1921), with a value of $k / \phi=3.4 \pm 0.2 \times 10^{-10} \mathrm{~m}^{2}$. The maximum rise height of liquid was measured to be $2.7 \mathrm{~cm}$, which corresponds to $p_{s} \approx-264 \mathrm{~Pa}$.

To make the sponges effectively two-dimensional, OHP transparencies were glued onto thin slices of the sponge, as shown schematically in figure 3. This prevents water from flowing except through the thin open edges. To investigate how different solid densities affect the time taken to sink, we taped various metal weights to the base of the sponge without impeding the fluid flow into the sponge. The object was then placed in 

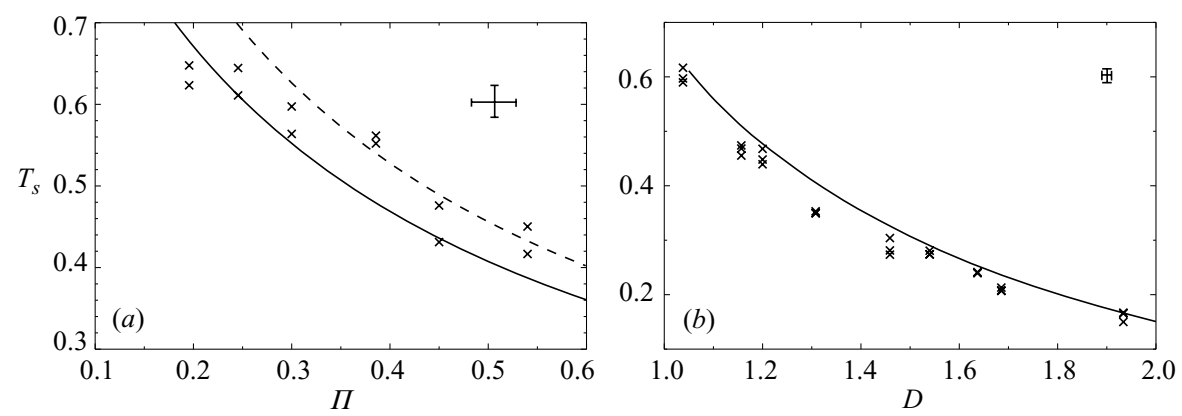

FIGURE 4. The time taken to sink in different experiments (crosses) compared to our theoretical predictions (curves). (a) Dependence of $T_{s}$ on the strength of surface tension $\Pi$ for a one-dimensional sponge with $D=1.65 \pm 0.05$. Curves show the analytical prediction $(2.10)$ with $D=1.6$ (dashed) and $D=1.7$ (solid). (b) Dependence of $T_{s}$ on solid density for a two-dimensional sponge with $\Pi \approx 0.42$ and $\alpha \approx 2.1$. Curve shows the corresponding prediction from boundary integral simulations. In experiments $\phi \approx 0.77$. Typical error bars are shown in the top right-hand corner of each plot.

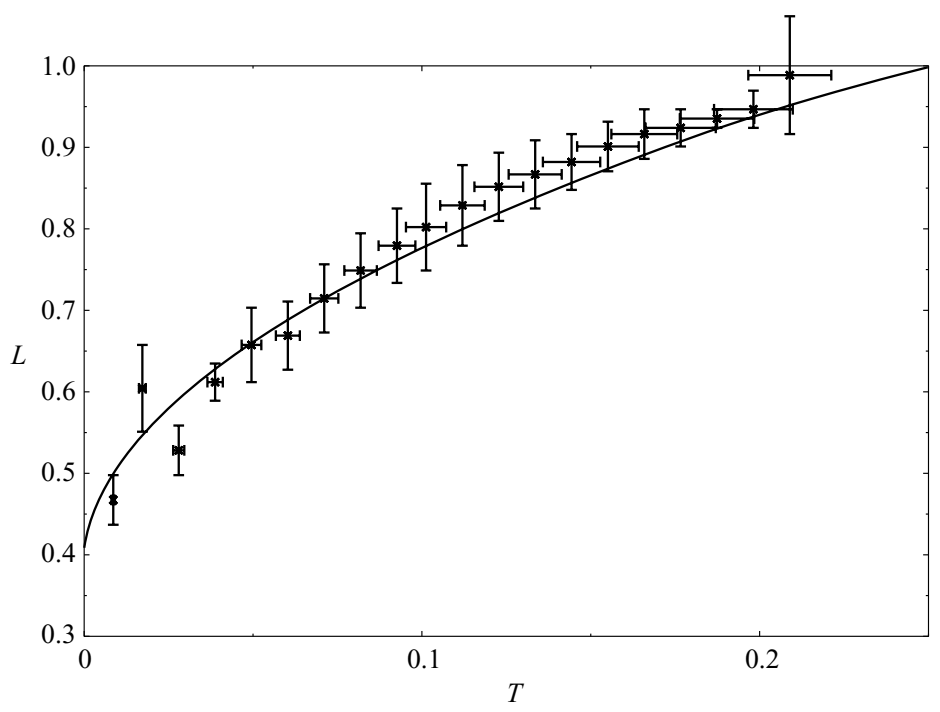

FIGURE 5. The time evolution of the non-dimensional submerged body depth. Experimental results (points) compare well with the theoretical prediction (solid line). Here $\phi=0.77, D=1.64$, $\Pi=0.42$ and $\alpha=2.1$.

a bath of water and the time taken for it to sink measured. The presence of a weight at the base of the object also stabilized the object to toppling, ensuring that it remained upright throughout the experiment. In another series of experiments, we sealed the vertical edges of a two-dimensional sponge to make it effectively one-dimensional. In the one-dimensional experiments, we varied $\Pi$ by varying the height of the sponge.

The results of our experiments, together with the relevant theoretical predictions, are presented in figure 4 . These show that there is good agreement between experiment and the theoretical predictions. We note that in the case of the two-dimensional object (figure $4 b$ ), the theory systematically overpredicts the time taken for the object to sink. This may be attributed to the early time transients in the experiment where the object is not initially in hydrostatic equilibrium. As can be seen from a graph of its level in the water (figure 5) the object initially oscillates about hydrostatic equilibrium. 
During these oscillations the object soaks up water faster than the model suggests: it initially falls below its equilibrium depth where the hydrostatic pressure is relatively high, forcing liquid in faster. This increases the weight of the object and it does not rise as far during its upward motion as it would with its initial weight. For the one-dimensional object (figure $4 a$ ), waterlogging takes longer so these early transients are less significant and the experimental results agree with theoretical predictions to within the experimental errors.

\section{Discussion}

Our experimental and theoretical analyses have shown how the time taken for a porous object to sink depends on its size and material properties. The dependence of sinking time on the size of the object is particularly relevant in sedimentology. According to Manville et al. (1998), pumiceous sediments that formed at the bottom of a body of water are unusual in being 'inverted': that is they consist of smaller pumice fragments at the base of the sediment with increasing fragment size towards the top. Manville et al. (1998) suggest that this could be explained by the smaller pumice fragments becoming waterlogged more quickly than larger fragments and so reaching the bed earlier. They explained this using an analogy between the motion of liquid in a porous medium and diffusion and predicted that, in our notation, $t_{s} \sim d^{2}$. The results of our numerical simulations, summarized by the scalings in (3.5), show that this is correct for fragments that are small in comparison to the equilibrium capillary rise height in the material. For larger fragments, however, the liquid motion is driven primarily by the hydrostatic pressure head in the surrounding liquid so that $t_{s} \sim d$. This difference in exponents will not alter the postulated size 'inversion' of pumiceous deposits. However, it will be valuable in estimating the time taken to sink, an important quantity in other contexts, as we now discuss.

There are many recorded instances in which long-lived pumice 'rafts' have formed on open water shortly after volcanic eruptions, perhaps the most famous being the Krakatoa eruption of 1883. More than six months after the eruption, individual pumice fragments as well as larger 'pumice rafts' were washed up on the east coast of Africa (Symonds 1888). The vast distances covered by pumice in this and other instances has led to the suggestion that floating pumice and other material could act as a means of biological dispersal carrying plants and even small animals between distant continents (McBirney \& Williams 1969); field studies by Jokiel (1989) have shown that marine organisms (including coral colonies) are commonly brought into the Kwajalein Atoll (Marshall Islands) onboard floating pumice.

For biological dispersal via pumice to be feasible, pumice fragments must typically remain afloat for long periods of time. While this longevity has been observed numerous times in the field, there are no quantitative theoretically based estimates of it. In general, this will depend on the strength of surface tension in driving the flow. However, from figure 2 we see that $T_{s} \leqslant O(1)$ for all values of the surface tension strength $\Pi$. Given the non-dimensionalization of time in (2.3), we therefore expect that

$$
t_{s} \leqslant O\left(\frac{d \phi \mu}{k \rho_{l} g}\right) .
$$

Klug \& Cashman (1996) found that pumice typically has $k \gtrsim 10^{-14} \mathrm{~m}^{2}$, which then suggests that $t_{s} \leqslant O(1)$ day for $d=1 \mathrm{~cm}$. However, the experiments of Manville et al. (1998) and Whitham \& Sparks (1986) both show that pumice fragments of this size actually float for $O\left(10^{3}\right) \mathrm{hr} \approx O(40)$ days before sinking. This discrepancy is 
interesting because our own experiments with pieces of sponge found good agreement between experiment and theory. An obvious explanation for the discrepancy is that the pore spaces within the pumice might be disconnected, thereby inhibiting the flow of water between them. However, Whitham \& Sparks (1986) appear to have ruled out this possibility by soaking some of their pumice specimens in molten wax, allowing it to solidify and then dissecting the rock to check that the pore spaces had been infiltrated. Alternatively, the discrepancy may illustrate the importance of inhomogeneities in the porosity and permeability of pumice, which have not been characterized experimentally.

We are grateful to Mark Hallworth for discussions and to Anja Slim for bringing this problem to our attention. D. V. is supported by Trinity College, Cambridge. The research of H.E.H. is partially supported by a Royal Society Wolfson Merit Award.

Note added in proof. After this article had been accepted for publication we became aware of Thornham \& Wilson (1983) on the settling of a porous body through a density interface between two fluids. This situation is qualitatively similar to that studied here, though they neglect the interfacial tension and assume that interfacial deformations remain small throughout the motion, and obtain different results.

\section{REFERENCES}

Batchelor, G. K. 1967 An Introduction to Fluid Dynamics. Cambridge University Press.

BeAR, J. 1988 Dynamics of Fluids in Porous Media. Dover.

Bryan, S., Cook, A., Evans, J., Colls, P., Wells, M., Lawrence, M., Jell, J., Greig, A. \& Leslie, R. 2004 Pumice rafting and faunal dispersion during 2001-2002 in the southwest pacific: record of a dacitic submarine explosive eruption from Tonga. Earth Planet. Sci. Lett. 227, $135-154$.

Corless, R. M., Gonnet, G. H., Hare, D. E. G., Jerey, D. J. \& Knuth, D. E. 1996 On the Lambert $W$ function. Adv. Comput. Math. 5, 329-359.

Greenhow, M., Vinje, T., Brevig, P. \& Taylor, J. 1982 A theoretical and experimental study of the capsize of Salter's duck in extreme waves. J. Fluid Mech. 118, 221-239.

JoKIEL, P. L. 1989 Rafting of reef corals and other organisms at Kwajalein Atoll. Mar. Biol. 101, 483-493.

Klug, C. \& Cashman, K. V. 1996 Permeability development in vesiculating magmas: Implications for fragmentation. Bull. Volcanol. 58, 87-100.

Longuet-Higgins, M. S. \& CoKelet, E. D. 1976 The deformation of steep surface waves on water. I: A numerical method of computation. Proc. R. Soc. Lond. A 350, 1-26.

Manville, V., White, J. D. L., Houghton, B. F. \& Wilson, C. J. N. 1998 The saturation behaviour of pumice and sedimentological implications. Sediment. Geol. 119, 5-16.

McBirney, A. R. \& Williams, H. 1969 Floating pumice as a means of biological dispersion. Mem. Geol. Soc. Am. 118, 113-116.

PhiLIP, J. R. 1969 Theory of infiltration. Adv. Hydrosci. 5, 215-305.

Symonds, G. J. 1888 The Eruption of Krakatoa and Subsequent Phenomena, Report of the Krakatoa Committee of the Royal Society. Harrison and Sons.

Thornham, S. A. \& Wilson, S. D. R. 1983 The settling of a porous body through a density interface. J. Fluid Mech. 126, 369-378.

Tsai, W.-T. \& Yue, D. K. P. 1996 Computation of nonlinear free-surface flows. Annu. Rev. Fluid Mech. 28, 249-278.

Washburn, E. W. 1921 The dynamics of capillary flow. Phys. Rev. 17, 273-283.

Whitham, A. G. \& Sparks, R. S. J. 1986 Pumice. Bull. Volcanol. 48, 209-223. 